Article

\title{
Comparative Agent-based Simulations on Levels of Multiplicity Using a Network Regression: A Mobile Dating Use-case
}

\author{
Joseph A.E. Shaheen ${ }^{1, \dagger * \mathbb{D}, \text { Collin Henley }}{ }^{2, \ddagger}$, Liam McKenna ${ }^{2,}$, Steven Hoang ${ }^{2,}$, and Fatma Abdulwahab ${ }^{2,}$ \\ 1 Georgetown University, Massive Data Institute; js822@georgetown.edu \\ 2 George Mason University \\ * Correspondence: js822@georgetown.edu; (J.A.E.S) \\ $\dagger$ McCourt School of Public Policy \\ \$ These authors contributed equally to this work.
}

\begin{abstract}
We report an agent-based model to compare the effectiveness of simple and complex mobile dating application interfaces in generating matches for virtual users. We define the relative complexity of dating applications as the number of available features and dub this variable, the multiplicity. We replicate some of the most popular mobile dating applications through the generation of a synthetic population endowed with attributes, preferences, and behaviors drawn from literature. We treat our data as a network dataset and use a robust statistical procedure (MRQAP) to issue a valid and reliable comparison between simulated applications. We show how the quadratic assignment procedure can be used to compare network simulations rigorously. As a result, we observe a direct relationship between multiplicity and agent-level experiences and expectations in match generation. We also observe the emergence of divergent matching systems with minor rule changes as well as several expected properties of online dating systems. This work serves as a proof-of-concept in the integration of classical social network analysis methods with agent-based modeling to compare virtual designs and to enhance the policy-generation process of online social networks.
\end{abstract}

Keywords: online dating, social networks, agent-based modeling, mobile dating applications, MRQAP

\section{Introduction}

Mobile dating applications have experienced dramatic growth in user adoption with 15 of the most popular commercial instances acquiring 247 million downloads in 2018 alone [1]. Currently, roughly $15 \%$ of all U.S. adults report using a dating app to find a mate. By 2021 the market focused on virtual matching is expected to reach \$3-\$5 Billion and year-over-year growth of 25\% [2]. The surge in mobile dating revenues is driven by several factors, including more accepting social norms and better accessibility. In a 2005 study conducted by Pew Research Center, 44\% of Americans indicated that "online dating is a good way to meet people" [3]. By 2013, 59\% agreed with this statement and $21 \%$ indicated that "online dating has lost much of its stigma."

Today's mainstream mobile dating applications offer common features and a market-driven (emerged) universal user interface, but they continue to vary in levels of multiplicity - that is - the number of features available to users in order to achieve some desired result. The desired result is often finding a suitable mate but not always [4]. Although previous research has explored several aspects of online dating e.g. recommender systems [5,6], online disinhibition [7,8], mate preferences [9-11], self-presentation [12-14], few works have tended towards a system-level agent-based modeling approach (see $[15,16])$.

\section{Background}

Tinder is one popular mobile dating applications partly due to its simple and user-friendly interface. This single application generates an estimated 15 million matches daily [2]. Once installed on a mobile device new users are prompted to create a profile to showcase themselves to potential matches. Profiles consist of images uploaded by the user and a single text-based input field for that can be used as a profile summary. More recently, a field in the user interface for "Interests" was also 
incorporated. Upon completion of the user profile users can choose matching preferences based on age, gender, distance, location and other attributes. Users sort sequentially through profiles of potential matches shown to them by the application's recommendation algorithm. Users can then perform an action to either accept or reject any given profile presented to them by the embedded algorithm. After each decision action has been made, a user is presented with a new profile and the acceptance/rejection process is repeated. Consequently, when two users 'like' each other, a notification is displayed. This is deemed a 'match' and users are now able to communicate directly in a private chat room.

As Tinder's popularity grew, other mobile dating applications emerged with their own features and interfaces. Hinge — another popular mobile dating application-allows users multiple pathways to match, extending the Tinder-like accept/reject functionality while providing more powerful search features. Hinge allows users the option to create more detailed profiles that specify ethnicity, political views, desire to have children, and other common attributes and preferences. Users can also enrich their profiles with clever prompts that enhance their appeal to visitors; profile visitors can 'like' these or comment on them. Like Tinder, when mutual likes occur users are then 'matched'. A key difference between Tinder-like applications and Hinge-like applications is the requirement that users mutually 'like' one another prior to activating communication features. Hinge-type applications allow users to send messages concurrently with 'like' actions if they desire, while Tinder-type applications require a match before communication can occur. This enables communication as an embedded feature in the 'like' request for applications similar to Hinge and allows for more specialized matching strategies. The ability to send a message with a like request differentiates Hinge-type from Tinder-type applications in important ways and creates more decision pathways to reach a match, which we dub, multiplicity. We argue that the multiplicity of dating applications plays a principal role in aggregate system outcomes and the overall experiences of users. Moreover, we argue that users can navigate the range of available applications to maximize their dating potential and match utility based on the advantages or disadvantages of the system-level multiplicity of considered applications. To that end, users who have not generated much interest when using Tinder-type applications can opt to utilize a user interface that allows for a more specialized matching strategy; perhaps one that places more emphasis on tailored personal messages rather than strict personal attributes (e.g. age, ethnicity, education). A more specialized strategy can only be undertaken on dating systems that allow for multiple pathways to matching-those with a higher multiplicity. Consequently, multiplicity could be directly correlated with match success for non-majority groups who must select a specialized mate-selection strategy often divergent from the mainstream.

In this paper, we construct principal models of two dating application agent-based simulations, one with a low multiplicity representing applications such as Tinder, and another with a higher level representing applications such as Hinge. We dub them Multiplicity Level 1 (M1) and Multiplicity Level 2 (M2), respectively with the lower rank (M1) representing Tinder-type applications and the higher rank (M2) reserved for Hinge-type applications. Both models were assigned rule-sets with the purpose of replicating their respective real-world counterparts. Our approach combines agent-based modeling and classical social network analysis as our go-to analytical frameworks. As outputs from our simulation models we construct networks containing various link types reflecting agent interactions through the simulated mobile dating application. We define a 'like' interaction as a one-sided, directed tie representing interest of a sender agent to some receiving agent, a 'match' as dyadic interest of two agents (two-directional 'like' links), a 'dislike' as a directed rejection, and a 'message' as a combination interaction (mechanistically, we model this as a 'like' with a higher probability of a response). Through implementation of numerous simulations we investigate the contribution of various mechanisms in generating matches.

This paper advances the state of the art in social network simulations in several ways. Primarily, the paper relies on social science theory to simulate an application's interface using well-studied social effects drawn from peer-reviewed literature in the absence of relevant data which were often unobtainable. Although this approach is not common in the social sciences it is necessary for our specific inquiry: Public datasets do exist but in two forms, each of which are either not useful or offer very limited use: The first is aggregate data from non-peer reviewed sources, much of which is not held to high empirical standards in collection or reporting, mitigating proper and standard 
evaluations of data integrity. Much of this data comes in the form of summary totals of likes and matches listed on Internet blogs by commercial entities. It has been shown that the connection between aggregate data sources and agent decision rule probabilities is generally non-linear and mathematically intractable [17] rendering such data sub-optimal for agent-based model development, and in the absence of complete aggregate datasets (joint distributions for example), we consider them to be insufficient for external validation and thus unusable. The second form-which we utilize in a stylized manner-is drawn from empirically reviewed sources (e.g. [18]), and this collection of data and models offer statistical agent attribute and behavioral insights into the mechanics of online mate selection. We consider them sufficient in offering insights towards our central question: How does the multiplicity of dating applications affect agent and aggregate matching outcomes? Thus, we make good and reasonable use of this collection.

\subsection{Homophily as a Framework}

We rely on homophily [19-22] as a primas inter pares foundational driver for our virtual experiments. We incorporate this sociological framework in our models-though admittedly-ancillary sociological processes contribute to the overall potential of matching and mate selection on online dating platforms. The principle of network homophily implies that "people's personal networks are homogeneous with regard to many socio-demographic, behavioral, and interpersonal characteristics" [23]. Homophily is a powerful social dynamic which often indicates that agents prefer those who are similar to themselves in many areas of social, economic, and cultural discourse. As a cognitive process, it is shown to be correlated with incentives for positive interactions resulting from shared knowledge, beliefs, and attributes. In the context of online dating, perceived similarity has been shown to correlate with positive attraction. [24] measure the effects of homophily in mate selection by sampling a pool of users on an online dating application and analyzing their preferences for potential partners in a range of categories. The authors analyze similarities such as desire for children, level of education, and physical appearance. They report that similarity increases the likelihood of a match. There is much evidence along this line of inquiry that support this finding [25? -28]. Specifically, we incorporate general notions of homophily in age, physical attractiveness, ethnicity and gender using a mix of discrete uniform categorical (ethnicity, gender) and continuous uniform (age, attractiveness) input attributes. Restricting our model to uniformity in the generation of agents' attributes provides a baseline of analysis and reduces the possibility that our conclusions are influenced by our agent population generative choices. Thus, homophily is a reasonable choice as the theoretical framework for model design.

\section{Materials and Methods}

\subsection{Principal Model Mechanics}

We use the Python module NetworkX [29] to build our model and run simulations. NetworkX is a module of the Python programming language that allows for the manipulation of network data and/or simulations through edge list objects or adjacency matrices. We use NetworkX's node functionality to represent the agents of our model, and the link functionality to represent interactions between the agents (likes or messages). ${ }^{1}$

To ease the process of comparing outputs from our models, the number of agent attributes were kept to a plausible minimum and kept consistent across both models. Agent populations were endowed with a binary gender (male and female), a generic ethnicity chosen from 4 categories, physical attractiveness (between 0 and 1), and age (18-65). All agent attributes were assigned uniformly. In both model variations (M1 and M2), we restrict population size to 1000 agents. To duplicate users' time constraints and in some instances online applications' fixed limits (Tinder fixes the number of likes per day that a user can generate under their basic plan), through every turn of the simulation agents are presented with no more than 40 agents of the other gender to consider. Dating applications will often present users with profiles within a set range as well and so agents in our models are only presented with those who are within 10 years of age difference. Finally, each agent

1 Our model code is published on the OpenABM initiative, CoMSES: https://www.comses.net/codebase-release/8378f5c840d7-4809-aa83-e45cdaf2f586/ 
Table 1. Assignment of agent attributes across all simulation models.

\begin{tabular}{c|c|c} 
Input & Variable Type & Assignment \\
\hline \hline Ethnicity $(\mathscr{E})$ & Discrete Uniform & $\mathrm{U}[0,1,2,3]$ \\
\hline Age $(\mathscr{A})$ & Continuous Uniform & $\mathrm{U}[18,60]$ \\
\hline Physical Attractiveness $(\mathscr{P})$ & Continuous Uniform & $\mathrm{U}[0,1]$
\end{tabular}

Table 2. Rules applied by agents for calculation of overall compatibility (total score). Compatibility is assigned as the additive accumulation of all assigned attributes (age, attractiveness, ethnicity). Second column conveys conditions under which the rule calculation is presumed. All value calculations and parameters are assigned such that they will produce a maximum partial score of 1 . Independent compatibility scores are then added to produce overall compatibility score. Description column contains parameter values chosen for a typical run. [Key: $\top:$ :(same as), $\perp$ :(different from)]

\begin{tabular}{|c|c|c|c|}
\hline Model Attribute & Condition & Value & Description \\
\hline $\begin{array}{l}\text { Compatibility Score } \\
\text { (ethnicity) }\end{array}$ & $\begin{array}{l}\mathscr{E}_{M} \top \mathscr{E}_{F} \\
\mathscr{E}_{M} \perp \mathscr{E}_{F}\end{array}$ & $\begin{aligned} S_{e} & =\left(\frac{\mathscr{X}}{2}\right)+0.5 \\
S_{e} & =\left(\frac{\mathscr{X}}{2}\right)\end{aligned}$ & $\mathscr{X} \in[0,1]$ \\
\hline $\begin{array}{l}\text { Compatibility Score } \\
\text { (age) }\end{array}$ & $\mathscr{P}==M$ & $\begin{array}{l}S_{M}^{\mathscr{A}}=1-(2)\left(\left|\mathscr{N}(\mathscr{A})_{c d f}\right|-0.5\right) \\
\mathscr{N}(\mathscr{A})_{c d f}=\frac{1}{2}\left[1+\operatorname{erf}\left(\frac{x-\mu}{\sigma \sqrt{2}}\right)\right]\end{array}$ & $\begin{array}{l}\mu=-3.996 \\
\sigma=3.317 \\
x=\mathscr{A}_{M}-\mathscr{A}_{F}\end{array}$ \\
\hline $\begin{array}{l}\text { Compatibility Score } \\
\text { (age) }\end{array}$ & $\mathscr{P}==F$ & $\begin{array}{l}S_{M}^{\mathscr{A}}=1-(2)\left(\left|\mathscr{N}(\mathscr{A})_{c d f}\right|-0.5\right) \\
\mathscr{N}(\mathscr{A})_{c d f}=\frac{1}{2}\left[1+\operatorname{erf}\left(\frac{x-\mu}{\sigma \sqrt{2}}\right)\right]\end{array}$ & $\begin{array}{l}\mu=2.046 \\
\sigma=2.906 \\
x=\mathscr{A}_{F}-\mathscr{A}_{M}\end{array}$ \\
\hline $\begin{array}{l}\text { Compatibility Score } \\
\text { (attractiveness) }\end{array}$ & & $\begin{array}{l}S_{M}^{\mathscr{P}}=1-(2)\left(\left|\mathscr{N}(\mathscr{P})_{c d f}\right|-0.5\right) \\
\mathscr{N}(\mathscr{P})_{c d f}=\frac{1}{2}\left[1+\operatorname{erf}\left(\frac{x-\mu}{\sigma \sqrt{2}}\right)\right]\end{array}$ & $\begin{array}{l}\mu=0 \\
\sigma=0.341 \\
x=\mathscr{A}_{M}-\mathscr{A}_{F}\end{array}$ \\
\hline Total Score & & $\begin{aligned} S_{\text {Total }}^{M} & =\beta_{1}^{M} S_{e}^{M}+\beta_{2}^{M} S_{M}^{\mathscr{P}}+\beta_{3}^{M} S_{M}^{\mathscr{A}} \\
S_{\text {Total }}^{F} & =\beta_{1}^{F} S_{e}^{F}+\beta_{2}^{F} S_{F}^{\mathscr{P}}+\beta_{3}^{F} S_{F}^{\mathscr{A}}\end{aligned}$ & $\begin{array}{l}\beta_{1}^{M}=0.3 \beta_{1}^{F}=0.4 \\
\beta_{2}^{M}=0.3 \beta_{2}^{F}=0.3 \\
\beta_{3}^{M}=0.4 \beta_{3}^{F}=0.3\end{array}$ \\
\hline
\end{tabular}

evaluates a compatibility score of the considered agent and records an appropriate 'like' or 'dislike' response based on heterogeneous thresholds.

A compatibility score is a measure based on the attributes of alters (considered agents) and the preferences of ego (the agent). Applying a basic threshold criteria, if a given compatibility score exceeds ego's compatibility threshold, the probability of a "like" is set to some maximum-allowed parameter value. If this threshold is not reached, the probability of a "like" is minimized. We chose probability parameters arbitrarily in the absence of data but relied upon intuition to guide their relative magnitudes. If assembled agents record a bi-directional "like", a "match" is recorded. This threshold assignment is consistent with social threshold models [30] and their dating counterparts [11]. We summarize all agent rule calculations in Formula 2.

\subsection{Agent Preferences}

Agents in our model favor homophily in ethnicity and attractiveness as referenced in [23], while holding differential preferences in age (male agents preferring younger female agents and female agents preferring older male agents). As agents are randomly selected in every turn of the model they will evaluate whether to like each other based on these attributes and a previously set 
threshold. Generally, threshold levels determine the likelihood of a like or dislike event occurring and are summarized in Figures 1 and 2.

Parameter estimates are drawn from several peer-reviewed sources from within the literature on online dating. To begin, male agents were given a Gaussian preference for younger female agents where the mean difference was 3.996 years, and female agents were given a preference for older males with a mean difference of 2.046 years [31]. The compatibility score is intended to ensure that maximum score contributed by age resides at this mean, and the greater the difference from this mean difference, the lower the contribution of age to the total compatibility score. Physical attractiveness and ethnicity are based on similar principles. As we have noted, agents evaluate ethnicity and attractiveness based on the concept of homophily with much higher scores attributed when two agents of the same ethnicity or a similar attractiveness interact.

We also utilized a weighing mechanism for both male and female agents as genders may value attributes differently when conjoint with more attributes. The weights are multiplied by the agent-agent interaction scores then summed to yield a total score. This total score is then compared to a threshold value to determine the occurrence of a like relation. In line with [32]'s study showing that males value physical features more than females, male agents were given a higher weight $\left(\beta_{3}^{M}=0.4\right)$ for attractiveness preference than for female agents $\left(\beta_{3}^{F}=0.3\right)$. [33] concludes that females place greater weight in finding a potential match within their own ethnicity and so we adjusted the weighing and contribution for ethnicity to account for this $\left(\beta_{1}^{F}=0.4, \beta_{1}^{M}=0.3\right)$. Finally, the weighing of scores on age by both genders remained the same $(0.3)$ as there was little evidence that the contribution to a total score was different from both genders even in light of different age preferences for males $(\mu=-4.0)$ than for females $(\mu=2.05)$.

Since our model is subject to theoretical matching system fundamentals [34], synthetic population structure and heterogeneity play an important role in model specification. The choice of male to female agent ratios could - if chosen unwisely — create unintended consequences and artifacts in our model. Thus, we rely on [3]: The author reports that Tinder's user base is roughly $60 \%$ male and $40 \%$ female. Though this statistic is a rough estimate drawn from non-peer-reviewed sources, therefore we consider it a guide (and not a rule) to our implementation and model specification. As a result, we chose population proportion parameters consistent with the overall notion (that there are many more males than females on dating applications) at a rate of $68 \%$ men and $32 \%$ women in line with the general principle of a 2:1 ratio.

Once agents are presented with a profile to evaluate they proceed with said evaluation $(S \in[0,1])$ against a pre-set parameter to determine whether they will like the considered agent(s). These threshold parameters are assigned arbitrarily in the absence of data but follow the following intuitive patterns:

- $\quad$ Female agents threshold for a 'like' are higher than male agents [35] [M1 \& M2].

- The probability of a 'like' when the compatibility threshold is not achieved $\left(S_{\text {compatibility }}<\right.$ $S_{\text {Threshold }}$ ) is not zero. It is dramatically reduced but a 'like' may still occur. [M1 \& M2]

- A 'like with a message' is still subject to the same aforementioned compatibility thresholds, but increases the probability of a like event. [M2]

All thresholds, probabilities, and decision junctures are summarized in Figures ??. And while threshold levels and probabilities are homogeneous, each agent's compatibility score for every other considered agent is heterogeneous. This ensures that agents' internal standard for acceptance of a potential mate are antecedents in maintaining individual and independent preferences in agent-agent interactions. To illustrate, consider a female agent $F_{1}$ with ethnicity $=\mathrm{E}$, age $=30$, and attractiveness $=0$ or $\{\mathrm{E}, 30,0\}$ who evaluates a male agent $M_{1}$ with attributes $\{\mathrm{E}, 32.046,0\}$. Given our model rules $F_{1}$ will calculate that $M_{1}$ 's compatibility with her is $0.4\left(0.5+\frac{x \in U[0,1]}{2}\right)+0.3(1)+0.3(1)$. If we assume that $x \in U[0,1]=0.5$, then $F_{1}$ will view her compatibility with $M_{1}$ as $0.3+0.3+0.3=0.9$ or $90 \%$. The M1 model specifies that since her compatibility score is greater than the designated threshold, 0.5 (Figure 1), then there is an $80 \%$ probability of a "like" occurring. If the compatibility score was less than 0.5 , the probability of a 'like' would be $30 \%$.

This previous example illustrates the general mechanics of the M1 model (e.g. Tinder) where a message may not be sent with each like interaction. In model M2's specification (e.g. Hinge) two key 
differences exist. Firstly, agents may choose to send a 'like' or a 'like with a message'. We assume that a like with a message increases the probability of a reciprocated like (we assume messages are positive and well-suited). Consequently, it should be evident that the second key difference between our specified M1 and M2 Models is that ego in M2 considers the like of the alter in their decision to like alter. This latter effect is known as reciprocity [36] and is a direct result of the difference in our hypothetical application's user interfaces and multiplicity-the difference being that applications such as Hinge (M2) allow users to attach a message with a like prior to matching, while applications such as Tinder (M1) do not. This forces users to evaluate incoming interactions not only based on the sender's attributes, but on the message attached to the interaction as well. Finally, we should report that we chose to specify that all decision points are Bernoulli tests in (U[0,1]) and that coefficients attached to our formulaic rules (Figure 1 ) scale the compatibility score to be within $[0,1]$ to ensure a one-to-one comparison scheme.

\section{Model Comparison}

As we have noted, our objective was to compare two models with varied multiplicity levels in terms of aggregate outcomes. We chose to focus our effort on matches that agents accumulate in each model. We carried out a formal inquiry of the statistical parameters and distributions of likes, messages, and matches as well as evaluated conditional distributions through a network regression-the Multiple Regression Quadratic Assignment Procedure-a suitable method for our analysis that accounts for our model's rule dependencies without inappropriate assumptions. While the literature on methods of network comparisons is copious [37], we chose MRQAP for its minimal assumptions and for its relative ease of interpretation-reducing output comparison to a comparison of the coefficients of a regression model.

The Multiple Regression Quadratic Assignment Procedure (MRQAP) is used to test for significance in an observed correlation where dependency between two or more dyadic relations may exist [38]. MRQAP relies on a non-parametric permutation-based test that preserves the integrity of the observed network structures to report confidence. This approach was originally developed by [39] to identify geographic clustering of diseases [38]. Mantel and colleagues noted that covariates in geographic datasets are highly co-dependent and typically not independently and identically distributed (i.i.d.) due to relational processes. Consequently, the covariates are unsuitable for regression models that assume independence of observations-a common example is the method of Ordinary Least Squares (OLS).

MRQAP has been developed and deployed as a mainstream network analysis tool. The procedure is particularly useful when calculating coefficient magnitudes and parameter estimates, and as a corollary, the strength of social effects-through permutations of network statistics. Since our synthetic population samples, rules, attributes, and mechanisms are not i.i.d., we relied on this standard social network analysis model to compare outputs from our simulations. We omit a comprehensive discussion of the nature of this method at this time, but direct the reader to aforementioned citations. However, to summarize the method's mechanics: MRQAP provides regression coefficients as does classical regression models (ordinary least squares), but its assumptions to not fall within the General Linear Model (GLM). Since we cannot guarantee some of the basic assumptions of OLS when considering relational data structures - assumptions such as bivariate normality or the independence of observations-MRQAP calculates coefficient estimates but permutes the dependent and independent variables on the structure of the network [40] yielding conditional p-values. Simply put, MRQAP answers: How likely is a model, given a randomization of the dependent variable on the network's structure?

As we have explained, MRQAP is well-suited for our comparative aims: Consider that M1 (Figure 1) emulates the simplest dating application interface-an example we repeatedly cite is Tinder. We begin our analysis by considering likes and matches as edges in a network (ties), and thus we frame our output analysis as a network analysis with edges within both simulations' code-base reflecting all temporal interactions through the application's interface as edges in a network. Our analysis is then conducted using the cross-sectional network emerged from the temporal interactions. Framing our analysis in this way allows for the use of MRQAP and its powerful permutation-based 
statistical assumptions while accounting for non-independence in our model with minimal interfering assumptions.

To compare M1 to M2, we consider their shared output; In our case the common output is matches. We ensure that inputs among the two models are identical for a one-to-one comparison. A synthetic population instantiated for M1 can apply M2's behavioral rules without loss of generality. We use ethnicity, age, physical attractiveness as input attributes, and likes as an input variable in the network regression model. In turn, if we observe a statistical difference in the models' parameter estimates, given that we have randomly generated synthetic populations in both models according to the same rules, then this difference must be due to the difference in agent behaviors between the two models and not due to the attributes of the agent population. In our case, the difference between M1 and M2 lies squarely in an increase in multiplicity. The M2 model allows for the sending of a message with a like and M1 does not-hence, reciprocity as a social effect. In Section 4 we present typical results from a representative run of 1000 nodes for both model types and Figure ?? contain visualizations of the agent decision processes in both simulations.

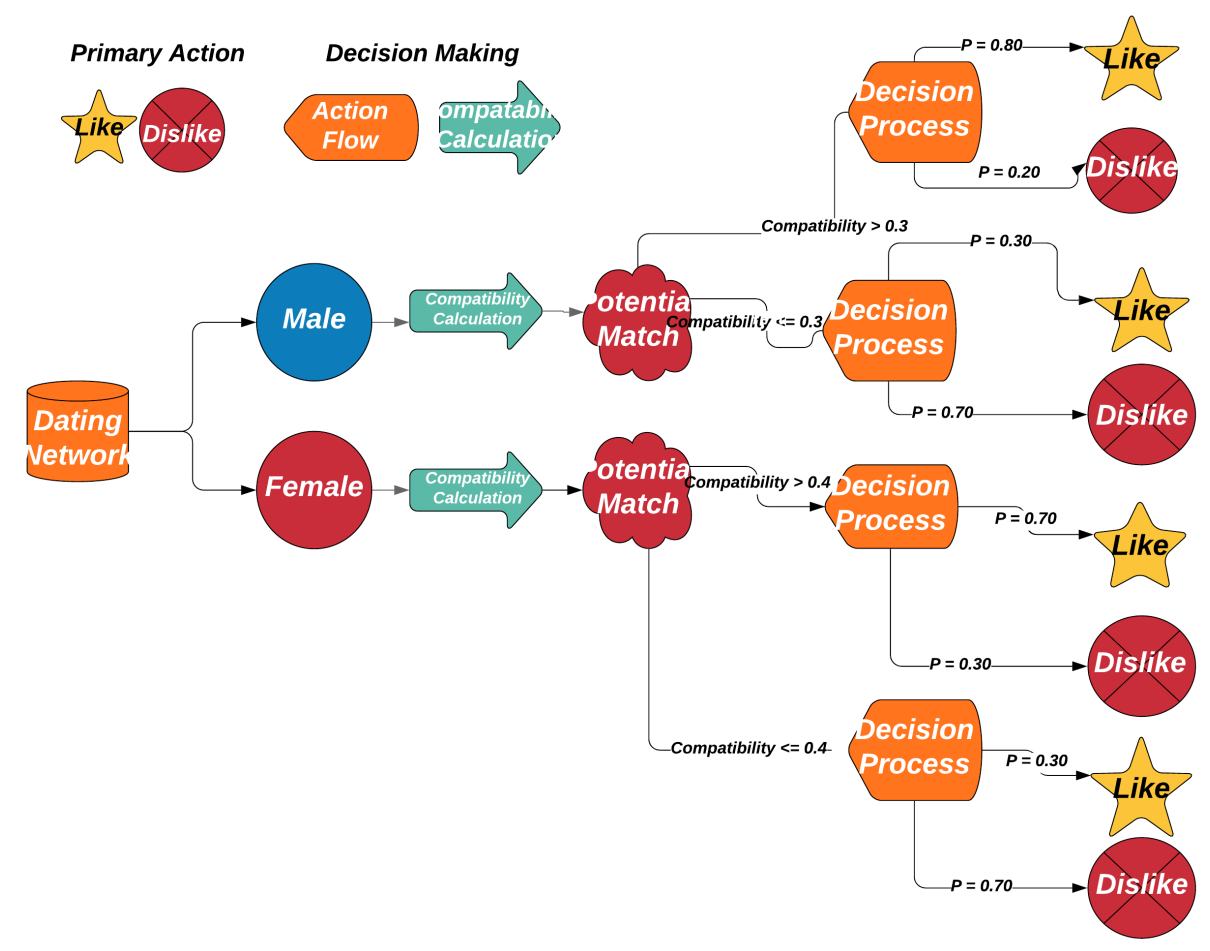

Figure 1. Main model multiplicity level one (M1) decision diagram for both male and female agents.

\subsection{Internal Validation}

Our primary process of internal validation was to ensure that all synthetic populations were generated to our strict requirements of uniformity for attractiveness [0,1], age [18-65], and ethnicity (categorical) [A, B, C, D]. Both tables ?? show the results of a representative comparison between generations of a synthetic population. The tables show agreement with our expectations and supply confidence that our code-base is reflective of our models' specifications. Since our goal is to quantify the difference of the models based on the given multiplicity of dating applications or the structure of this particular system, no further statistical validation of agent attributes was necessary since the outputs-given-structural-differences is the measure of comparison in this case.

To elaborate further, we would expect that the mean attractiveness for our population would be roughly 0.5 since we designated this variable as $U[0,1], \approx 42$ years of age as the mean value between 18 and 65 , and for ethnicity-a categorical variable-to be equally scaled among the $0^{\text {th }}, 25^{\text {th }}, 50^{\text {th }}$, and $75^{\text {th }}$ percentiles (lower bounds). This is clearly shown in our reported tables. 


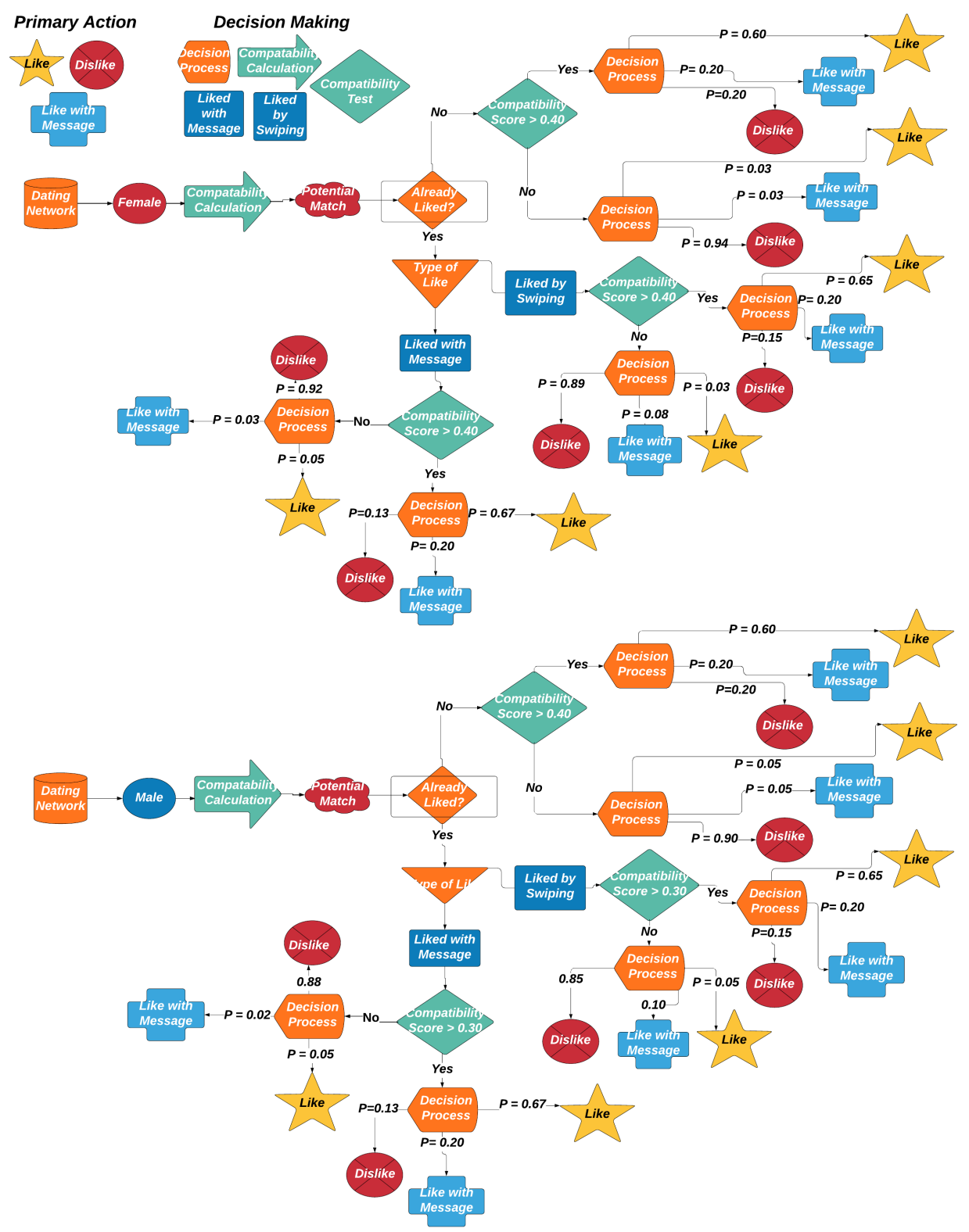

Figure 2. Main model multiplicity level two (M2) decision diagram for males (top) and females (bottom).

Table 3. Validation of simulation inputs for M1 model including type of statistic, mean, and quartiles

\begin{tabular}{lccccccc}
\hline \hline Statistic & $\mathrm{N}$ & $\mu$ & $\sigma$ & $0^{\text {th }}$ & $25^{\text {th }}$ & $75^{\text {th }}$ & $100^{\text {th }}$ \\
\hline Attractiveness & 1,000 & 0.512 & 0.293 & 0.001 & 0.257 & 0.778 & 0.999 \\
Age & 1,000 & 42.088 & 13.063 & 19 & 31 & 54 & 64 \\
Ethnicity & 1,000 & 2.462 & 1.122 & 1 & 1 & 3 & 4 \\
\hline
\end{tabular}

Table 4. Validation of simulation inputs for M2 model including type of statistic, mean, and quartiles

\begin{tabular}{lccccccc}
\hline \hline Statistic & $\mathrm{N}$ & $\mu$ & $\sigma$ & $0^{\text {th }}$ & $25^{\text {th }}$ & $75^{\text {th }}$ & $100^{\text {th }}$ \\
\hline Attractiveness & 1,000 & 0.483 & 0.284 & 0.001 & 0.229 & 0.723 & 1.000 \\
Age & 1,000 & 40.879 & 13.989 & 18 & 28 & 53 & 65 \\
Ethnicity & 1,000 & 2.495 & 1.101 & 1 & 2 & 3 & 4 \\
\hline
\end{tabular}




\section{Results}

5.1. Likes, Matches, and Messages
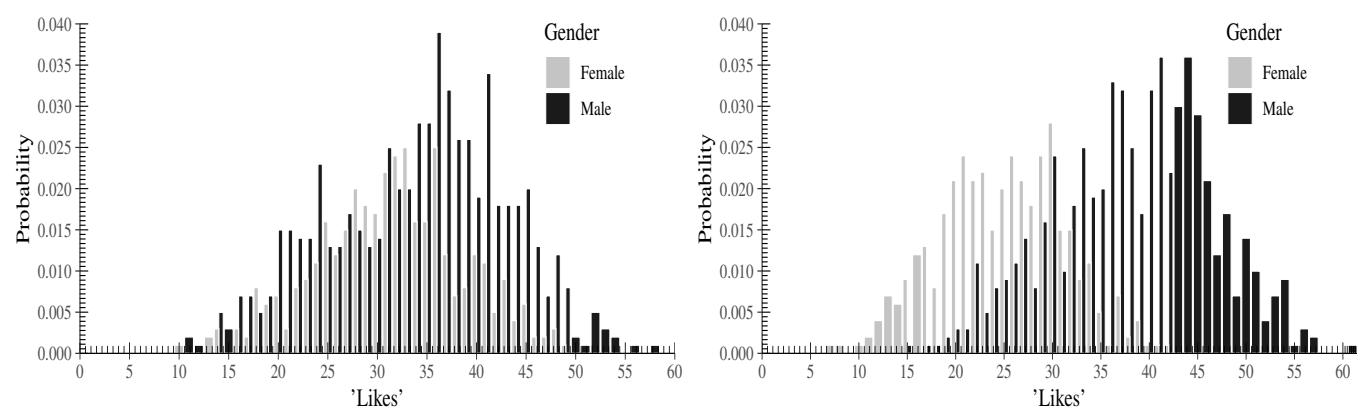

Fioure 4. Main simulation 'like' distrihutions for Multinlicitv I evel 1 (left) and Multinlicitv I evel 2 (Rioht)
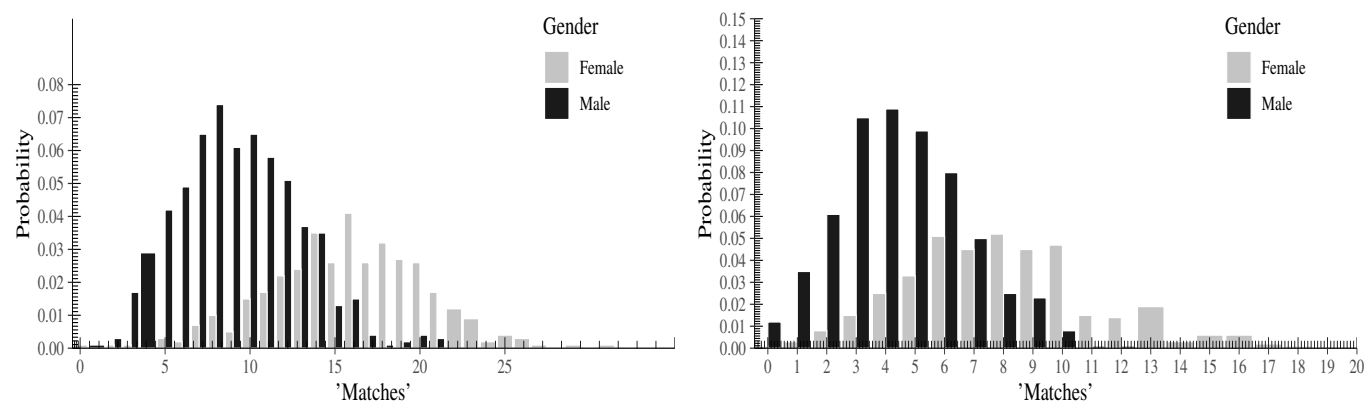

Figure 5. Main simulation 'match' distrihutions for Multinlicitv I evel 1 (left) and Multiplicity Level 2 (Right).
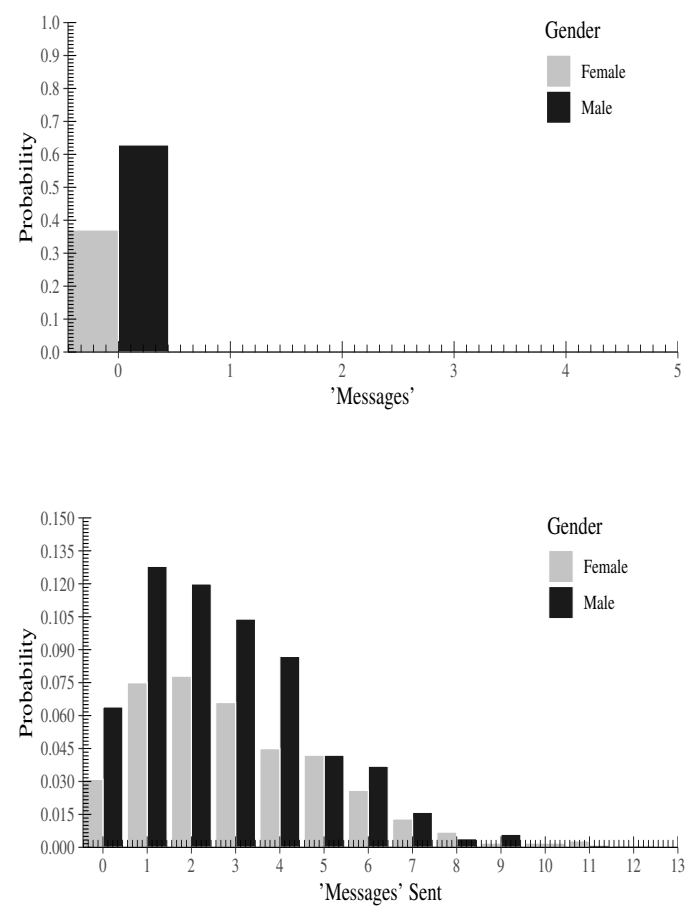

Figure 6. Main simulation 'message' distributions for Multiplicity Level 1 (top) and Multiplicity Level 2 (bottom). 
The general shape and location of the probability distributions of likes (Figure 4) was similar by design in both M1 and M2 with male agents generating slightly more likes than female agents. Male and female agents in both models liked and disliked other agents according to a bell-shaped distribution. This was anticipated and is likely a result of the use of Gaussian differential inputs in age and attractiveness. Because both models can essentially be viewed as matching processes, lower counts of female agents meant that male agents will 'like' more frequently given a fixed amount of activity per turn. Overall, there was no statistical difference in the total number of likes when comparing M1 to M2 without conditioning on gender. Table 7 shows the result of a paired t-test comparing the mean difference between statistics in M1 to M2. Excepting 'like' relations, which we designated to be equivalent in both simulations, we found statistical differences between the model types. As shown, M1 (e.g. Tinder) produced more likes, dislikes and matches but less messages (by design) than M2 (e.g. Hinge).

The similarity in the liking distribution however did not result in equiprobable matching distributions (Figure 5) either by gender or by model type. M1 model mechanics (Tinder) generated more aggregate matches for both male and female agents than M2 and as measured by median value. Dispersion was greater for both agent genders in M1 when compared to counterparts in M2 simulations. Female agents in both simulations received more matches than male agents by mean and median values. The probability distribution for female agent matches exhibited a longer tail, with top female agents receiving 3 times the number of matches of median males in M1 and 5 times the median male agents in M2. This implies a greater clustering of matches for female agents than for male agents. However, the median number of matches for male agents in both model types was much more probable than its female agent analogue with male agent median value in M1 representing 7\% of sample compared with $4 \%$ for female agents with $11 \%$ and $5 \%$ for $\mathrm{M} 2$ agents respectively.

Messaging distribution (Figure 6) analysis was relatively straightforward to consider since agents in M1 simulations did not possess the ability to send messages. M2 agents sent a median of 1 message for male agents and 2 messages for female agents and the messaging probability distribution carried a left skew with some agents sending more than 10 message per simulation run.

Tables ?? provide summary statistics for like, dislike, match and message distributions for a representative run in aggregate form and act as an additional internal validity test for simulation runs. Of central interest is the mean number of matches $(\mu)$ generated by M1 when compared to M2-almost precisely double. What is of additional significance is the variance reduction and shorter tail of M2. Specifically, the coefficient of variation for M1 is 0.42 while for M2 we calculate it as 0.53. This suggests a more even and equal distribution of matches among nodes-or in real-world terms, agents who were previously unsuccessful in receiving many matches in M1 receive more matches in M2, hinting towards the emergence of specialized strategies.

Table 5. M1 Model Run Summary Statistics including type of statistic, mean, standard deviation, and quartiles.

\begin{tabular}{lccccccc}
\hline \hline Statistic & $\mathrm{N}$ & $\mu$ & $\sigma$ & $0^{\text {th }}$ & $25^{\text {th }}$ & $75^{\text {th }}$ & $100^{\text {th }}$ \\
\hline 'Like' Count & 1,000 & 33.030 & 8.504 & 10 & 27 & 39 & 58 \\
'Dislike' Count & 1,000 & 146.600 & 60.624 & 48 & 105 & 207.2 & 264 \\
'Match' Count & 1,000 & 11.694 & 5.029 & 0 & 8 & 15 & 31 \\
'Message' Count & 1,000 & 0.000 & 0.000 & 0 & 0 & 0 & 0 \\
\hline
\end{tabular}

Table 6. M2 Model Run Summary Statistics including type of statistic, mean, standard deviation, and quartiles.

\begin{tabular}{lccccccc}
\hline \hline Statistic & $\mathrm{N}$ & $\mu$ & $\sigma$ & $0^{\text {th }}$ & $25^{\text {th }}$ & $75^{\text {th }}$ & $100^{\text {th }}$ \\
\hline 'Like' Count & 1,000 & 33.029 & 9.983 & 7 & 26 & 41 & 61 \\
'Dislike' Count & 1,000 & 142.699 & 53.134 & 55 & 108.8 & 198.2 & 251 \\
'Match' Count & 1,000 & 5.844 & 3.112 & 0 & 4 & 8 & 18 \\
'Message' Count & 1,000 & 2.874 & 2.086 & 0 & 1 & 4 & 12 \\
\hline
\end{tabular}


Table 7. Statistical comparison of M1 and M2 models using a paired t-test. Shown are the parameter difference $\Delta b$, the t-statistic, $\mathrm{p}$-value and confidence interval of the parameter estimate. This table shows no mean difference in the number of likes yet a statistically significant mean difference in the number of matches between M1 and M2.

\begin{tabular}{ccccc}
\hline \hline Relation Type & $\Delta b_{M}$ & $t$ & $p$ & $95 \%$ CI \\
\hline 'Like' & 0.001 & 0.002 & 1.00 & {$[-0.81,0.8]$} \\
\hline 'Dislike' & 3.901 & 1.53 & 0.12 & {$[-1.07,8.87]$} \\
\hline 'Match' & 5.85 & 31.35 & $\mathrm{p}<0.001$ & {$[5.48,6.21]$} \\
\hline 'Like Message' & -2.874 & -43.57 & $\mathrm{p}<0.001$ & {$[-3.00,-2.7]$} \\
\hline
\end{tabular}

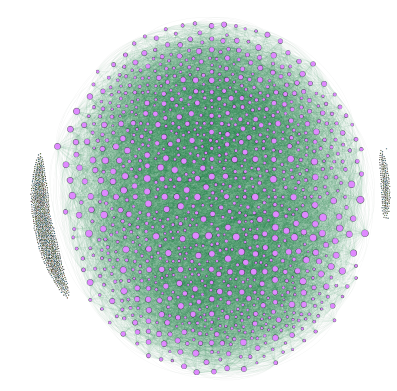

((a)) M1 'Like' Network.

((b)) M1 'Match' Network.

Figure 8. Match Network for typical simulation run for M1 model (Tinder)

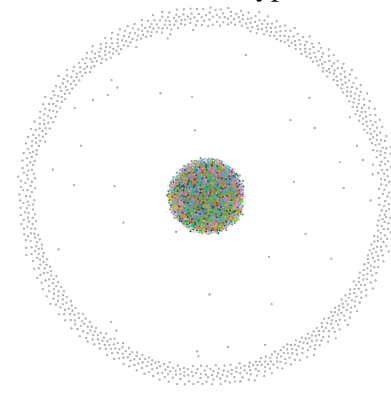

((a)) M2 'Like' Network. $\quad$ ((b)) M2 'Match' Network.

Figure 9. Match Network for typical simulation run for M2 model (Hinge).

\subsection{Regression Models}

By framing our outputs as networks of interactions between agents, the resultant 'like' and 'match' networks shown in Figures ?? gain significance. Since much of the mechanism of the underlying agent rules was intentionally stochastic so would be our outputs. Nevertheless, nonrandom structure is evident and it can be presumed as having emerged from model rules. M1's match network produced a large component and many isolates, indicating that small preferences (in age for example) can produce clusters of nodes where there is high matching probability. The M2 match network showed highly dyadic structure (rather than transitive, as in in M1) suggesting that agents matched with one or two other agents, but not within larger clusters of agents as in M1.

To ensure a principled comparison, we utilized the network regression technique-MRQAP [39]-to investigate the relationship of our monadic and dyadic covariates. MRQAP requires that we convert node attributes such as attractiveness, age, and ethnicity into difference (or similarity) adjacency matrices. Intended as a one-to-one comparison, the models in Table 8 included the 'like' network, attractiveness difference, age difference and ethnicity difference as independent/predictor variables and the 'match' network as the dependent variable. We consider the effects of covariate inputs on producing a match as an output given the multiplicity of our two model types. 
Table 8. Multiple regression model of monadic and dyadic covariates using the quadratic assignment procedure. One model for Tinder-type applications (M1) and two models for Hinge-like applications (M2) are shown. The Message relation covariate is only included in the second model of the M2 model for comparison. Reported are the parameter estimates and p-value estimates for each effect. $\operatorname{Pr}(<=)$ conveys the proportion [0,1] of networks generated/permuted with identical structure to the observed network with a parameter value less than or equal to the reported estimate. $\operatorname{Pr}(>=)$ conveys the reverse with the proportion of permuted networks with a parameter value greater than observed. $\operatorname{Pr}(>|=|)$ reveals symmetrical properties of the parameter distribution with nil indicating perfect symmetry and other values indicating skewness.

\begin{tabular}{|c|c|c|c|}
\hline & M1 & M2 (False) & M2 (True) \\
\hline like network & $2.55 \mathrm{e}-01$ & $-6.05 e-03$ & $-6.06 e-03$ \\
\hline $\operatorname{Pr}(<=b)$ & $(1.0)$ & $(0.0)$ & $(0.0)$ \\
\hline $\operatorname{Pr}(>=b)$ & $(0.0)$ & $(1.0)$ & $(1.0)$ \\
\hline $\operatorname{Pr}(>=|b|)$ & $(0.0)$ & $(0.0)$ & $(0.0)$ \\
\hline message network & & & $-4.35 \mathrm{e}-03$ \\
\hline $\operatorname{Pr}(<=b)$ & & & $(0.0)$ \\
\hline $\operatorname{Pr}(>=b)$ & & & $(1.0)$ \\
\hline $\operatorname{Pr}(>=|b|)$ & & & $(0.0)$ \\
\hline attractiveness (difference) & $2.04 \mathrm{e}-04$ & $-6.87 e-04$ & $1.64 \mathrm{e}-04$ \\
\hline $\operatorname{Pr}(<=b)$ & $(0.8)$ & $(0.93)$ & $(0.92)$ \\
\hline $\operatorname{Pr}(>=b)$ & $(0.2)$ & $(0.07)$ & $(0.01)$ \\
\hline $\operatorname{Pr}(>=|b|)$ & $(0.45)$ & $(0.14)$ & $(0.1)$ \\
\hline age (difference) & $2.02 \mathrm{e}-07$ & $1.64 \mathrm{e}-04$ & $-4.96 \mathrm{e}-06$ \\
\hline $\operatorname{Pr}(<=b)$ & $(0.55)$ & $(0.01)$ & $(0.01)$ \\
\hline $\operatorname{Pr}(>=b)$ & $(0.45)$ & $(0.99)$ & $(0.99)$ \\
\hline $\operatorname{Pr}(>=|b|)$ & $(0.99)$ & $(0.03)$ & $(0.03)$ \\
\hline ethnicity (difference) & $1.24 \mathrm{e}-04$ & $-4.00 \mathrm{e}-06$ & $2.14 \mathrm{e}-04$ \\
\hline $\operatorname{Pr}(<=b)$ & $(0.70)$ & $(0.78)$ & $(0.8)$ \\
\hline $\operatorname{Pr}(>=b)$ & $(0.30)$ & $(0.22)$ & $(0.2)$ \\
\hline $\operatorname{Pr}(>=|b|)$ & $(0.65)$ & $(0.38)$ & $(0.42)$ \\
\hline intercept & $3.15 \mathrm{e}-03$ & $5.88 \mathrm{e}-03$ & $5.90 \mathrm{e}-03$ \\
\hline $\operatorname{Pr}(<=b)$ & $(1.0)$ & $(1.0)$ & $(1.0)$ \\
\hline $\operatorname{Pr}(>=b)$ & $(0.0)$ & $(0.0)$ & $(0.0)$ \\
\hline $\operatorname{Pr}(>=|b|)$ & $(0.0)$ & $(0.0)$ & $(0.0)$ \\
\hline Permutation(s) & 100 & 100 & 100 \\
\hline $\mathrm{R}^{2}$ & 0.1809 & 0.000205 & 0.000218 \\
\hline Adjusted $\mathrm{R}^{2}$ & 0.1809 & 0.00020 & 0.00021 \\
\hline Residual Std. Error $(\mathrm{df}=998995)$ & 0.097 & 0.25 & 0.076 \\
\hline F Statistic $(\mathrm{df}=4 ; 998995)$ & 55170 & 51.24 & 43.59 \\
\hline
\end{tabular}

Note:

models are statistically significant at the $p<0.0001$ level 
Reported are three models of interest-a single model describing M1 simulation results and two models for M2 simulation runs, the first of which is a model without an effect for messaging-a false model-the second of which (M2-T) includes the 'message' network as an independent variable. Of note are the order of magnitude for all parameter estimates, which tend towards remarkably small values. For example, the M1 like relation estimate is 0.255 ; Assuming variable independence and given in absolute terms, it took roughly 4 likes to produce a match for M1 agents. This is in line with expectations about the density of the like network when compared to the density of the match network-the former expected to be more dense and the latter more sparse.

Confidence in the reported parameter estimates is conveyed by the proportion of network permutations that meet lower-end one-tailed $\operatorname{Pr}(>=)$, upper-end one-tailed $\operatorname{Pr}(<=)$, and two-tailed tests $\operatorname{Pr}(>|=|)$. These reports communicate important features of the simulated systems. For example, $100 \%$ of the ensemble of networks permuted by M1 regression resulted in parameters for the like relation that were smaller $(<=b)$ ) than the observed effect size of 0.255 and that were larger than $(>=b))-0.0061$ for M2-F and -0.0061 for M2-T. This represents high confidence that this parameter differs significantly from a random observation of the same structural network. Also reported is a two-tailed measure of confidence $(\operatorname{Pr}(>|=|))$ expressing the skewness of the sampling distribution of estimates.

The 3 models presented in Table 8 explore a true model for M1 with all available monadic and dyadic effects, a false model for M2 (M2-F) without the messaging network, and a second model for M2 with messaging included. It was clear that all models are significant when compared to random. M2-F generated a model with a much higher standard error (0.25) when compared with M1 (0.097) and the true M2-T model (0.076). The coefficient of determination for the M1 model $\left(R^{2}=0.18\right)$ was greater than both M2 model equivalents, given that the number of agent decision points needed to achieve a match are more numerous in the M2 model regressions. Each of these decision points are subject to additional (uniform) random draws. This increases the amount of uniform random noise in simulation runs but leaves the reliability of the models intact.

Table 9. Parameter estimates ordered by contribution to match network (left compartment) from large values to smaller values (including negative values), and by magnitude rank (overall contribution). e.g. while the like relation for the M2-T model was a top contributor to the match dependent variable (rank by magnitude $=1$ ), it was also a negative parameter estimate placing it 5 th in the order of contribution.

\begin{tabular}{lcccc||ccc}
\hline & \multicolumn{3}{c}{ Order of Contribution } & \multicolumn{2}{c}{ Rank by Magnitude } \\
\hline & M1 & M2-F & M2-T & M1 & M2-F & M2-T \\
\hline like network & 1 & 4 & 5 & 1 & 1 & 1 \\
message network & - & - & 4 & - & - & 1 \\
attractiveness (difference) & 2 & 3 & 2 & 2 & 2 & 2 \\
age (difference) & 4 & 1 & 3 & 3 & 2 & 3 \\
ethnicity (difference) & 3 & 2 & 1 & 2 & 3 & 2 \\
intercept & $\mathrm{X}$ & $\mathrm{X}$ & $\mathrm{X}$ & $\mathrm{X}$ & $\mathrm{X}$ & $\mathrm{X}$
\end{tabular}

Beyond model-level reliability and validity our virtual experiment provides agreeable results. We rank coefficient estimates in Table 9 by order of contribution (from positive estimates to negative estimates) and by coefficient size (magnitude). Here we are observant of the coefficient estimates and the direction and distribution of likely parameters generated by the permutations of the quadratic assignment procedure. That is-we are interested in rank order (of appearance), magnitude (absolute value), and the ensemble of networks with an exact structure to our observed network. Figure 11, Table 8, and Table 9 summarize these quantities.

The M1 regression model produces a straightforward linear system where more 'like' activity produces more matches. This is due to a positive intercept, positive like parameter estimate with a valid test statistic extant on the upper tail $(\operatorname{Pr}(b<=))$, and greater differences in attractiveness. 
We find that only $55 \%$ of networks produced higher estimates than observed. Primarily, this is due to the systemic differences in how male and female agents evaluate age with males preferring slightly younger females and females preferring slightly older males. As a consequence, estimates are centered in a range that describe opposing positive and negative difference in age. By rank order of contribution, the like variable is the largest contributor to matches, followed by attractiveness, ethnicity, and then age. Ranked by magnitude, the like relation is the primary contributor to matches, while attractiveness and ethnicity contribute along the same rank, and age contributes (in absolute terms) roughly 5 times less than whether an agent likes another of an ideal difference in age versus 3 or more standard deviations away, placing it 3rd in magnitude rank.

It was a useful exercise in model validity to report two models for the M2 dating system-one which included the messaging network (M2-T) and one which omitted it (M2-F). The first clue in the non-applicability of M2-F's regression model is the relatively high residual standard error (0.25) when compared to the true model (0.076), an order of magnitude of difference. The non-inclusion of the messaging network as an independent variable caused the quadratic assignment procedure to shift the (positive) estimate for attractiveness and ethnicity to a negative estimate and assigned the parameter estimate for ethnicity a much smaller weighing (from $10^{-} 6$ to $10^{-} 4$ ). Under conditions where this data may have been gathered from a real-world sample one may be inclined to accept these differences at face-value, but because we know that a proper model must be inclusive of the messaging network-included in M2-T- since the behavior was incorporated in the simulation's specifications, we know that it must be false. The greater clue comes from the rankings of effects by magnitude (Table 9) which shows that M1 and M2-T have emerged the same order of effect ranking-that is-the variables contribute similar amount to the creation of a match for agents, while M2-f conveyed a differing order.

Greater confidence in M2-T as a valid model begins with the standard error of the model, which was roughly equivalent to M1 (0.076, 0.097 respectively). M2-T produced estimates for the like and messaging networks that were strong by rank (first) followed by attractiveness and ethnicity (second) and finally, age (third). This ranking of parameter estimates for M2-T is identical to M1 with excepting the messaging network rank which is not applicable in the case of the M1 model. The order of contribution was divergent however; While the like network was the strongest predictor of a match in the M1 regression model by (positive value, by order 1st), the like network was the weakest predictor of a match in the M2-T model (negative value, by order 5th). Agents in M2 could not rely on 'liking' alone to generate a match-in fact, liking many profiles resulted in less matches overall. This is a direct result of the additional multiplicity of the model, i.e. the addition of the messaging feature. Interestingly, attractiveness (by order, 2nd) held its order position in both models. Age was more relevant in M2 (by order, 3rd) than in M1 (by order, 4th) and messaging did not exist in M1 but was ranked 4th in M2, by rank order.

The third component of our findings was the likelihood function of parameter estimates describing the ensemble of networks permuted by MRQAP. Figure 11 summarizes those results through a direct comparison of the 3 models. The intercept likelihood sub-Figure 11 uniquely represents the divergence of Tinder-like applications and Hinge-like applications with intercept estimates for the M2 dating system far exceeding M1. This indicates that M2 agents initialize with a higher likelihood of matching-holding all else equal-but due to negative parameter estimates for liking and messaging must be more tactical with liking and messaging decisions than M1 agents. This hypothesis is uniformly confirmed through an increased variance of estimated parameters for all M1 (Tinder-like model) variables and increased certainty of estimates in both M2-F (false Hinge-like model) and M2-T (true Hinge-like model) estimates. In general, M2 parameter estimates for the 100 permuted networks under consideration were more likely and in some cases (age and ethnicity) were twice as likely as their M1 counterparts. The increased likelihood and less variant distribution of parameter estimates for the M2 system specifies a narrower corridor of conditions that must be met in order for matches to occur and as a consequence agents should employ specialized strategies in messaging. This finding is robust despite that agents in either simulation models are zero-intelligence (see [41]). 
Intercept

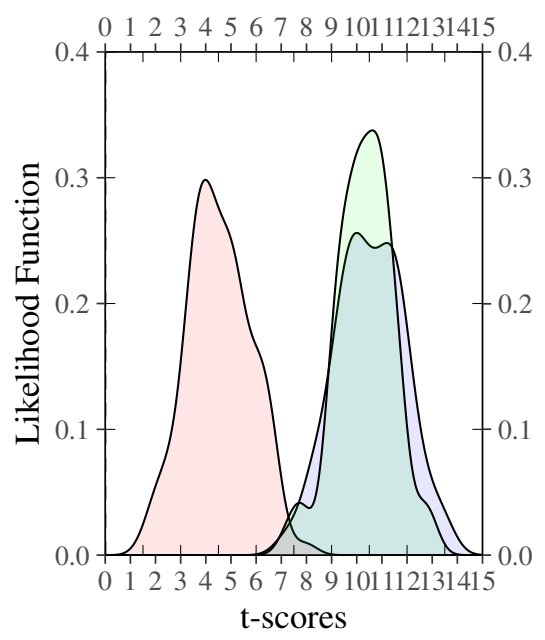

Message Parameter Estimate

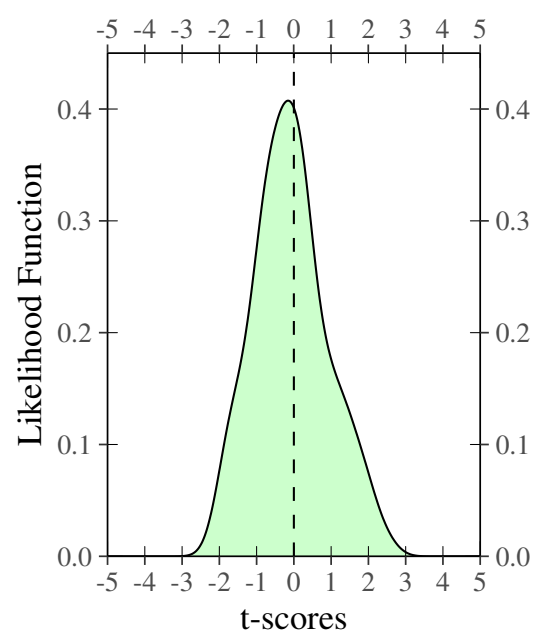

Age Parameter Estimate

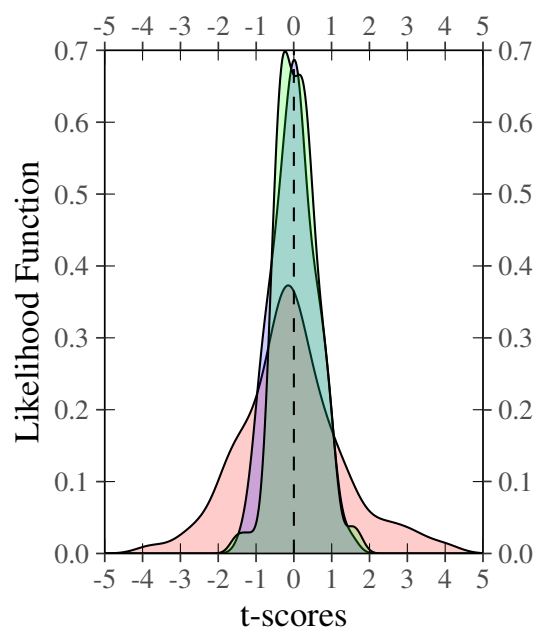

Like Parameter Estimate

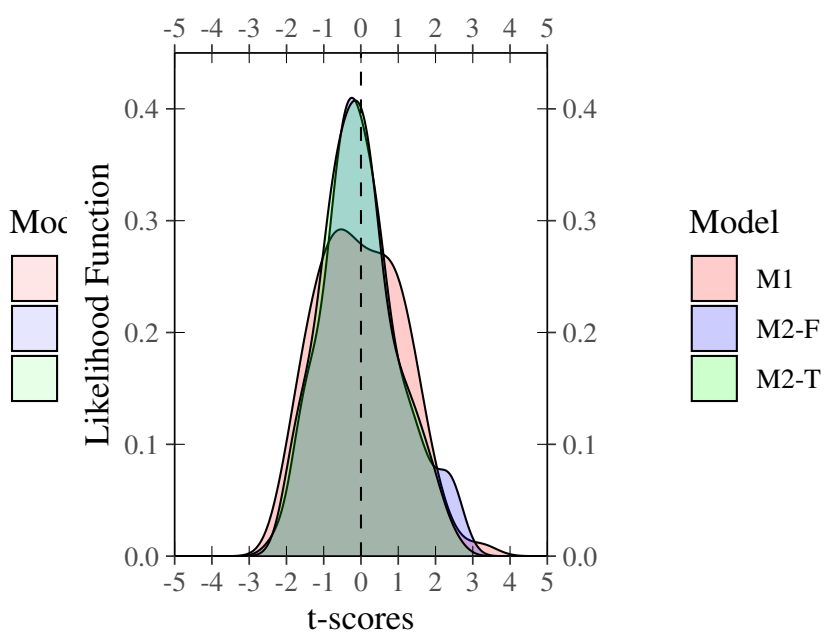

Attractiveness Parameter Estimate

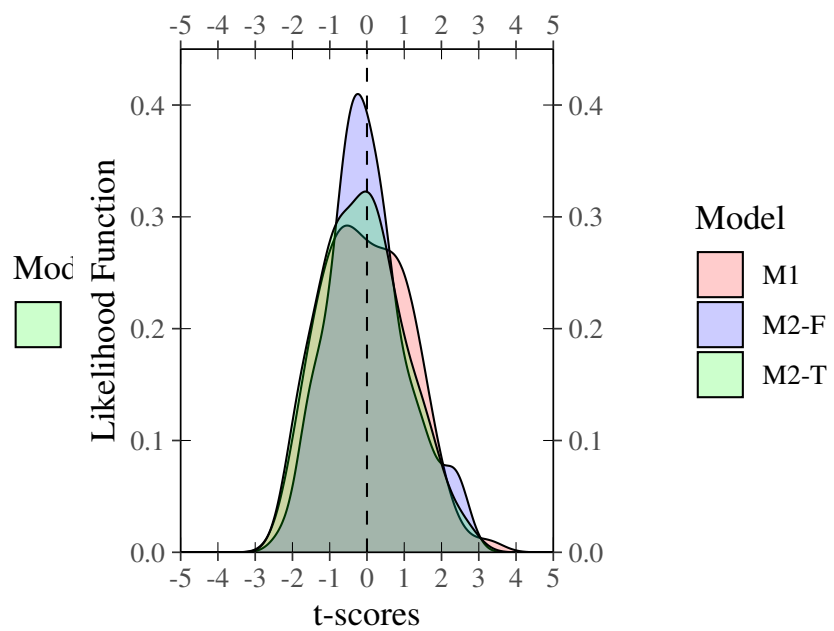

Ethnicity Parameter Estimate

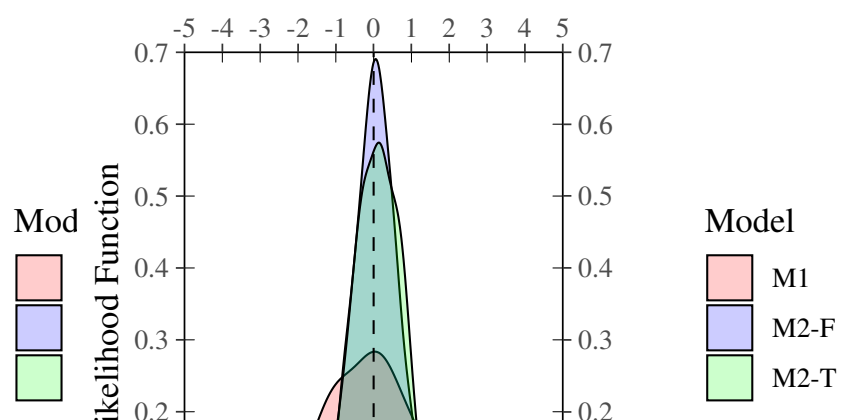

Figure 11. Likelihood functions for each parameter estimate by standardized scores drawn from Quadratic Assignment Procedure regression permutations. 


\section{Sensitivity Analysis}

To ensure that our results were independent of our parameter choices, we tested an elementary adaptation of each model simulation using a single agent rule. Agents in this base model evaluate the overall attractiveness of other agents through the uniformly assigned physical attractiveness attribute. The attractiveness score is then adopted as the overall score rather than combined additively with other scores. Analysis of the base model helps to determine the likelihood of bias due to design artifacts in our model. Similarly to our previous approach, we simulated both a Tinder-like application (M1) and a Hinge-like application (M2). Since our aim is not to compare our simulations to extant data but to each other, this step is not wholly necessary but is useful for model validity.

Figure 13 shows a comparison of M1 and M2 model run outputs and upholds our summary findings from previously reported model. The figures show no significant difference between stylized outputs of the previous model and this more basic version. Tables ?? provide summary statistics. Of note is the almost identical gender-based like and match probability distributions to the reported model in Figures ??. Despite the aggregation of compatibility scores without age or ethnicity playing a role, the results were a reflection of the main model reported as our central results with one exception: agents in the M1 base model generated more matches in the aggregate than their counterparts in the main results model previously reported. Since this M1 model is a simple matching system without diversity of conditions, this was to be expected.

Table 10. M1 sensitivity model run summary statistics including type of statistic, mean, standard deviation, and quartiles

\begin{tabular}{lccccccc}
\hline \hline Statistic & $\mathrm{N}$ & $\mu$ & $\sigma$ & $0^{\text {th }}$ & $25^{\text {th }}$ & $75^{\text {th }}$ & $100^{\text {th }}$ \\
\hline 'Like' Count & 1,000 & 11.365 & 3.900 & 1 & 9 & 14 & 26 \\
'Dislike' Count & 1,000 & 146.581 & 61.168 & 49 & 108 & 203.2 & 268 \\
'Match' Count & 1,000 & 12.810 & 5.558 & 0 & 9 & 16 & 32 \\
'Message' Count & 1,000 & 0.000 & 0.000 & 0 & 0 & 0 & 0 \\
\hline
\end{tabular}

Table 11. M2 sensitivity model run summary statistics including type of statistic, mean, standard deviation, and quartiles

\begin{tabular}{lccccccc}
\hline \hline Statistic & $\mathrm{N}$ & $\mu$ & $\sigma$ & $0^{\text {th }}$ & $25^{\text {th }}$ & $75^{\text {th }}$ & $100^{\text {th }}$ \\
\hline 'Like' Count & 1,000 & 32.875 & 9.645 & 10 & 26 & 40 & 61 \\
'Dislike' Count & 1,000 & 140.707 & 56.232 & 50 & 103 & 200 & 251 \\
'Match' Count & 1,000 & 5.682 & 3.036 & 0 & 3 & 7 & 16 \\
'Message' Count & 1,000 & 2.841 & 2.068 & 0 & 1 & 4 & 14 \\
\hline
\end{tabular}

\section{Discussion}

Both M1 and M2 simulations produced a right-skewed 'like' distribution with many agents receiving a small number of likes while a small number of agents receiving a disproportionately larger number of likes. Under the generally uniform choices we imbued agents with, we would not expect that outputs be heavy-tailed. There is strong evidence of skewness from peer-reviewed literature in [11] where the authors show a right-skewed distribution of message interactions that yield a match and this is also directly confirmed in [18]. Our own finding that many agents received few matches while a minority received many matches is confirmed by the finding in [11] as well. Furthermore, [18], a study conducted on Tinder profiles and interaction data provides the relative proportion of likes and matches, showing that the number of likes compared to the number of matches is larger by 2 or 3 orders of magnitude, consistent with Tables ??.

Though we surmise that skewness-as a system property-likely depends on the scale of dating platforms (e.g. the number of active subscribers) it is likely that if the number of agents were to be larger by an order of magnitude or more, then by sheer chance alone, the proportion of 

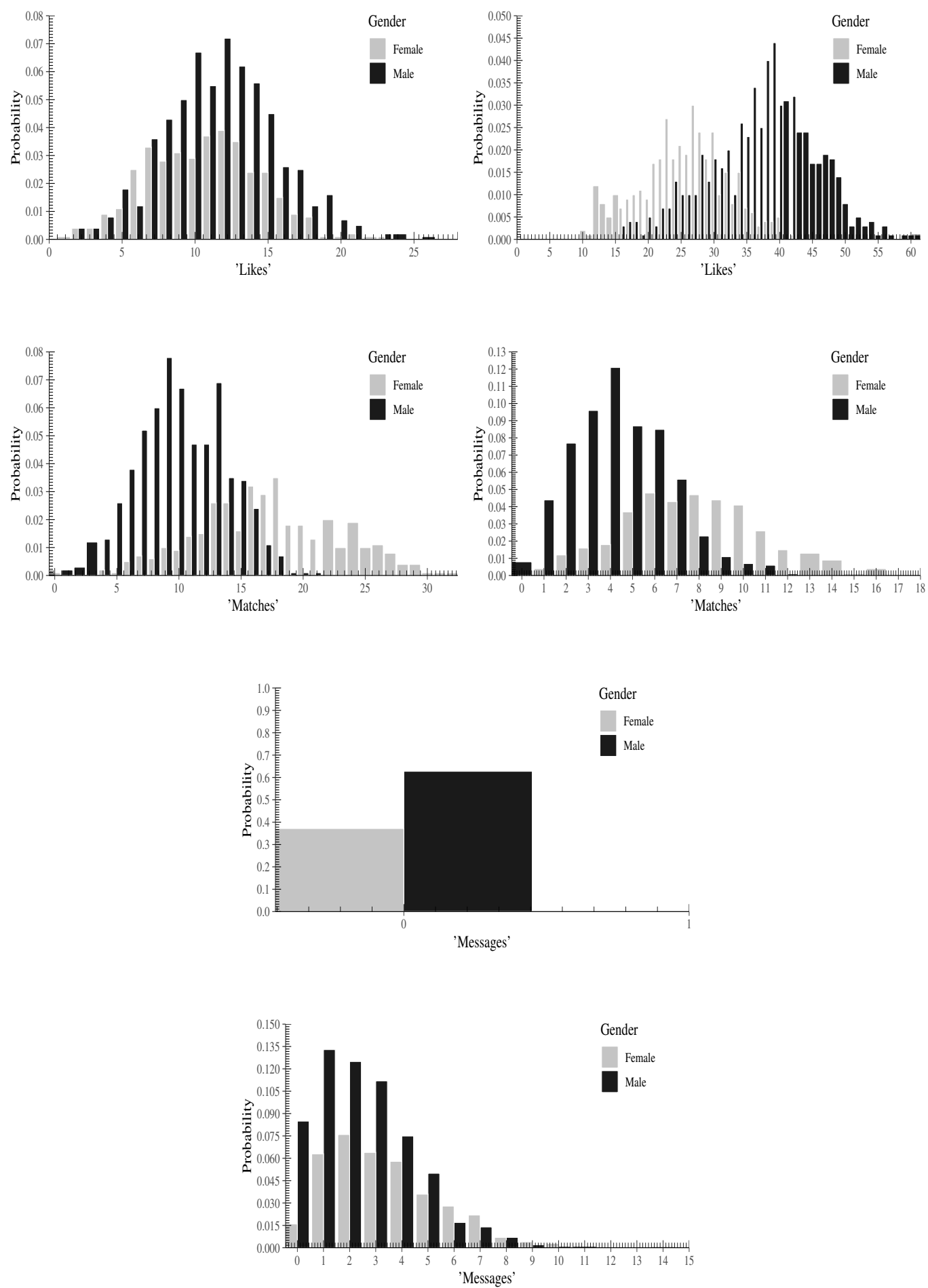

Figure 13. Sensitivity model summary with direct comparisons between M1 (left) and M2 (Right) variation runs including 'like' network (top left/right), 'match' network (middle left/right), and 'message' network probability distributions (middle top/bottom). 
agents receiving no matches would be higher-as top agents would continually gather a larger share of all available matches. Nominally, whether the well-studied scale-free (power law degree distribution) property [42] is an adage of our system is an interesting theoretical question that arises here. With ever-larger scale (Tinder is larger in membership count than Hinge) would the skewness and consequently the tails of our 'like' statistical distribution approach some extreme-value and heavy-tailed habitat that represents some biased social process. In the case of the classical scale-free property, the underlying social process is generally taken to be preferential attachment when the dataset under question is analyzed as a network. Though this question provokes a revision to our consideration of skewness as being a sufficiently reproducible stylized fact, it is assumed that even under conditions of preferential attachment (that produce highly skewed, heavy tailed output distributions), there will exist some internal balancing act (algorithmic) by dating service providers to ensure that-for example-an entire city does not subsequently match with a single user and that no single user is to receive unlimited matches. Thus, our assumption of skewness without extending said assumption to require outputs to be of an extreme value nature seems realistic and sufficient in the absence of additional data.

Figures ?? show that male agents received and sent more likes than female agents while female agents received more matches and messages than male agents while sending less messages than male agents. This reproduced collection of facts is directly confirmed by [43]. More male agents populated dating services [11] and also many more male agents were likely to receive no likes, matches, or messages than female agents [44]. This was also reproduced by our reported agent-based model.

Focusing our attention on the parameter estimates of our observed networks from M1 and M2-T, we see the effect of gaining additional multiplicity given in the form of an additional feature to send messages with likes. We found that the both M1 and M2-T simulations produced positive estimates for the intercepts and M2-T produced negative estimates for the like and message networks as well as the age difference covariate. One interpretation of the change in sign is that the M1 regression model describes a positively increasing regression model where more activity by M1 agents (more right swipes) helped agents produce more matches, contrasting M2-T decreasing-slope regression model where M2 agents could not simply increase their activity levels to increase the likelihood of a match. That is-agents engaging in non-strategic activity produced less matches overall in M2-T. The implications are profound: Agents, when presented with additional features, must employ strategies that reflect the availability of features and often these strategies must be more specialized. M2 agents were presented with a random sample of other agents for consideration and their messaging behavior, for the sake of comparison, was also assigned randomly. If we were to assign messaging behavior to be in line with total compatibility scores it would endow M2 agents with strategic behavior, and yet the regression models revealed this as a condition of success without the additional endowment. [45,46] discuss examples of online daters integrating more specialized behaviors in line with what we have produced.

Additionally, consider that for the M1 and M2-T models, the ranking of estimates remained invariant to the increase in multiplicity (Table 9). With both M1 and M2-T ranking the like network first concurrent with the messaging network (for M2-T), then attractiveness difference and ethnicity difference, and finally age difference, while simultaneously describing divergent system behaviors where more (random) likes imply a better chance of a match in the M1 system but did not in the M2-T system. We can conclude that while we produced the same systems in terms of effect importance, we also produced a clear divergence in their overall behaviors. Doubtless, effects are transformed when the option for reciprocity through an increased multiplicity is considered in M2-T by order (from positive to negative for example) but not by rank.

The overall divergence in aggregate system behavior is further demonstrated by the likelihood of parameter estimates generated by the quadratic assignment procedure and shown in Figure 11, clearly demonstrating that most parameter estimates for M1 and M2-T had similar properties excepting the intercept parameter estimate. Furthermore, M1's likelihood estimates were more variable as denoted by a wider functional form.

The interpretation of our combined results is based on two key indicators: The first is that the order of importance by coefficient effect size changed between M1 and M2-T-but not by rank 
order-even when given similar agent rules. The second is that we have successfully reproduced divergent system-level behavior in line with what would be expected from a pure matching market (Tinder) in comparison to a market where specialized strategies (e.g. messaging) can be adopted by agents. Remarkably, we did so by considering only the multiplicity of the applications rather than advanced psychological or sociological theories - a zero intelligence approach.

While we have confirmed our hypothesis, we must acknowledge there are limitations to our interpretations and consequently to our approach. The first of which is our use of agent attributes that did not conform precisely to what is known about dating application subscribers. For example, we use age intervals that we chose uniformly from a specified range, and for our main comparison we used ethnic identities that were evenly assigned. It is reasonable to assume that neither of these attribute assignment choices are realistic but were merely convenient. However, these choices were intended to be uninformed [47] in order to ensure that the attributes of agents cannot interfere in our statistical comparisons. Our goal herein was to issue a comparison of the relative size of parameter estimates not to estimate the parameters themselves. If we had chosen non-uniform attributes, then there exists a possibility that model artifacts would disturb our model comparisons. As a result, isolating the results due to a differing multiplicity would be more difficult.

While our method does achieve our intended results, in time a better methodological comparison may be possible. It should be noted that a comparison of datasets from two independent collections of observations is trivial to conduct in numerous ways. One need only consider the scientific inquiry in the statistics book of knowledge available since Gosset's t-statistic (1876) to find an overwhelming number of procedures that can be used to compare one set of observations to another. However, this is not what is being considered here; Here we are considering network effects as a representation of one dynamical system and attempting a comparison with a slightly different dynamical system through those same network effects-a less intuitive challenge. Nonetheless, the comparison yielded results and a robust conclusion.

\section{Conclusions}

In this paper, we have shown how agent-based simulations and a robust statistical method of comparison can be used to examine additional features of a dating application. Through this effort we have have discovered that inclusion of even one additional feature can cause divergent outputs and aggregate outcomes. We described this addition as increasing the multiplicity of an online dating application. We argued that this changes a user's personal experience and end-results: In this instance, overall matches and the underlying social effects that govern matching dynamics were transformed and we have emerged the basic properties of Tinder-like and Hinge-like dating application systems in a clear and demonstrable way. The M1 and M2 simulations models legislate clear differences in agent behaviors and in-turn their personal experiences. From this proof-of-concept, bridging our model to large-scale data and real world applications is not improbable and could enhance the design and development of more successful dating applications and matching environments for users. Surely, many who desire love would praise this effort.

As we conclude our report, it is most opportune to discuss an inherent assumption in our overall analysis which is best included along with our concluding remarks. This assumption is that "many who desire love", as we have dubbed them, desire quantity of matches as an important output, and seemingly as the output of choice. Consider [48]'s superb analysis of a sample of Tinder users: In this study of over 160 active Tinder users, an exploratory factor analysis reveals that out of six strong categories that explain users" behavior on Tinder, finding "love" was as important as finding "casual sex". In fact, the sub-scale of the analysis openly divulges that "to have a one-night stand" $(0.808)$ exceeded the explanatory strength of "to find a romantic relationship" (0.807). Importantly, from all six and including the four remaining categories (ease of communication, self-worth validation, thrill of excitement, and trendiness), self-worth validation "was the only motivation that was significantly related to higher Tinder use". Minimally, one can safely assume that while quality of match is always an intended target, because dating platforms have become a versatile collection of virtual meeting spaces used in different ways by different users hoping for different outcomes, all said outcomes are centered on the provision of more viable mating strategies - with an emphasis on 'more' rather than 'viable'. Not considering quality of matches, thus, can be described as a limitation of our approach 
only when assuming that "love" is the only important factor governing mating behavior on online platforms.

Henceforth, there are many directions we could pursue along this line of inquiry, including calibrating our social effects, models, inclusion of weighted dyadic relationship - and most importantly - using some reference dataset. The latter represents our work's greatest limitation since much of this data is considered private by commercial entities. We hope to be able to pursue these avenues in future papers, nonetheless.

\section{Author Contributions:}

Funding: not applicable

Institutional Review Board Statement: not applicable

Informed Consent Statement: not applicable

Data Availability Statement: Model code is provided at https://www.comses.net/codebase-release/8378f5c840d7-4809-aa83-e45cdaf2f586/

Acknowledgments: The methodological portions of this research were supported by an appointment to the Intelligence Community Postdoctoral Research Fellowship Program at Georgetown University for author (1).

Conflicts of Interest: No conflicts of interest.

\section{Abbreviations}

The following abbreviations are used in this manuscript:

MRQAP Multiple Regression Quadratic Assignment Procedure

\section{References}

1. Koch, L. Dating App Growth Slows, but Advertisers Shouldn't Ignore Hopeful Singles. https://www.emarketer.com/content/dating-appgrowth-slows-but-advertisers-shouldnt-ignore-hopeful-single-users, 2019.

2. Lin, M. Online Dating Industry: The Business of Love. https://www.toptal.com/finance/business-model-consultants/online-dating-industry, 2019.

3. McGrath, F. What to Know About Tinder in 5 Charts. https://blog.globalwebindex.com/trends/what-to-know-about-tinder/, 2015.

4. Zytko, D.; Grandhi, S.A.; Jones, Q. Frustrations with Pursuing Casual Encounters through Online Dating. Proceedings of the 33rd Annual ACM Conference Extended Abstracts on Human Factors in Computing Systems; ACM: Seoul Republic of Korea, 2015; pp. 1935-1940. doi:10/gkqbnk.

5. Brozovsky, L.; Petricek, V. Recommender System for Online Dating Service. Technical report, 2007, [cs/0703042].

6. Pizzato, L.; Rej, T.; Chung, T.; Koprinska, I.; Kay, J. RECON: A Reciprocal Recommender for Online Dating. RecSys'10 - Proceedings of the 4th ACM Conference on Recommender Systems, 2010, pp. 207-214. doi:10.1145/1864708.1864747.

7. Suler, J. The Online Disinhibition Effect. Cyberpsychology and Behavior 2004, 7, 321-326. doi:10.1089/1094931041291295.

8. Ellison, N.; Heino, R.; Gibbs, J. Managing Impressions Online: Self-Presentation Processes in the Online Dating Environment. Journal of Computer-Mediated Communication 2006, 11, 415-441. doi:10.1111/j.1083-6101.2006.00020.x.

9. Miller, G.F.; Todd, P.M. Mate Choice Turns Cognitive. Trends in Cognitive Sciences 1998, 2, 190-198. doi:10.1016/S1364-6613(98)01169-3.

10. Simão, J.; Todd, P.M. Emergent Patterns of Mate Choice in Human Populations. Artificial Life 2003, 9, $403-417$.

11. Hitsch, G.J.; Hortaçsu, A.; Ariely, D. What Makes You Click? Mate Preferences in Online Dating. Quantitative Marketing and Economics 2010, 8, 393-427. doi:10.1007/s11129-010-9088-6.

12. Hancock, J.T.; Toma, C.L. Putting Your Best Face Forward: The Accuracy of Online Dating Photographs. Journal of Communication 2009, 59, 367-386. doi:10.1111/j.1460-2466.2009.01420.x.

13. Hall, J.A.; Park, N.; Song, H.; Cody, M.J. Strategic Misrepresentation in Online Dating: The Effects of Gender, Self-Monitoring, and Personality Traits. Journal of Social and Personal Relationships 2010, 27, 117-135. doi:10.1177/0265407509349633.

14. Guadagno, R.E.; Okdie, B.M.; Kruse, S.A. Dating Deception: Gender, Online Dating, and Exaggerated Self-Presentation. Computers in Human Behavior 2012, 28, 642-647. doi:10.1016/j.chb.2011.11.010.

15. Simão, J.; Todd, P.M. Modeling Mate Choice in Monogamous Mating Systems with Courtship. Adaptive Behavior 2002, $10,113-136$. doi:10.1177/1059-712302-010002-03.

16. Shiba, N. Analysis of Asymmetric Two-Sided Matching: Agent-Based Simulation with Theorem-Proof Approach. Journal of Artificial Societies and Social Simulation 2013, 16, 1-14. doi:10.18564/jasss.2232.

17. Epstein, J.M. Agent-based Computational Models and Generative Social Science. Complexity 1999, 4, 41-60.

18. Tyson, G.; Perta, V.C.; Haddadi, H.; Seto, M.C. A First Look at User Activity on Tinder. Proceedings of the 2016 IEEE/ACM International Conference on Advances in Social Networks Analysis and Mining, ASONAM 2016 2016, pp. 461-466. doi:10.1109/ASONAM.2016.7752275. 
19. Hills, T.; Todd, P. Population Heterogeneity and Individual Differences in an Assortative Agent-Based Marriage and Divorce Model (MADAM) Using Search with Relaxing Expectations. Journal of Artificial Societies and Social Simulation 2008, 11.

20. Aral, S.; Muchnik, L.; Sundararajan, A. Distinguishing Influence-Based Contagion from Homophily-Driven Diffusion in Dynamic Networks. Proceedings of the National Academy of Sciences 2009, 106, 21544-21549, [1408.1149]. doi:10.1073/pnas.0908800106.

21. Reagans, R.E. Close Encounters: Analyzing How Social Similarity and Propinquity Contribute to Strong Network Connections. Organization Science 2011, 22, 835-849. doi:10.1287/orsc.1100.0587.

22. Centola, D. The Social Origins of Networks and Diffusion. American Journal of Sociology 2015, 120, 1295-1338. doi:10.1086/681275.

23. McPherson, M.; Smith-Lovin, L.; Cook, J.M. Birds of a Feather: Homophily in Social Networks. Annual Review of Sociology 2001, 27, 415-444. doi:10.1146/annurev.soc.27.1.415.

24. Fiore, A.T.; Donath, J.S. Homophily in Online Dating: When Do You like Someone like Yourself? Conference on Human Factors in Computing Systems - Proceedings, 2005, pp. 1371-1374. doi:10.1145/1056808.1056919.

25. Ibarra, H. Homophily and Differential Returns: Sex Differences in Network Structure and Access in an Advertising Firm. Administrative Science Quarterly 1992, 37, 422. doi:10.2307/2393451.

26. Gibbons, D.; Olk, P.M. Individual and Structural Origins of Friendship and Social Position among Professionals. Journal of Personality and Social Psychology 2003, 84, 340-351. doi:10.1037/0022-3514.84.2.340.

27. Klofstad, C.A.; McDermott, R.; Hatemi, P.K. The Dating Preferences of Liberals and Conservatives. Political Behavior 2013, 35, 519-538. doi:10.1007/s11109-012-9207-z.

28. Griffin, E.M.; Fingerman, K.L. Online Dating Profile Content of Older Adults Seeking Same- and Cross-Sex Relationships. Journal of GLBT Family Studies 2018, 14, 446-466. doi:10/ghenp5.

29. Hagberg, A.A.; Schult, D.A.; Swart, P.J.; Varoquaux, G.; Vaught, T.; Millman, J. Exploring Network Structure, Dynamics, and Function Using NetworkX. Proceedings of the 7th Python in Science Conference (SciPy2008); , 2008; pp. 11-15.

30. Granovetter, M. Threshold Models of Collective Behavior. The American Journal of Sociology 1978, 83, 1420-1443.

31. Conway, J.R.; Noë, N.; Stulp, G.; Pollet, T.V. Finding Your Soulmate: Homosexual and Heterosexual Age Preferences in Online Dating. Personal Relationships 2015, 22, 666-678. doi:10.1111/pere.12102.

32. Greenlees, I.A.; Mcgrew, W.C. Sex and Age Differences in Preferences and Tactics of Mate Attraction: Analysis of Published Advertisements. Ethology and Sociobiology 1994, 15, 59-72. doi:10.1016/0162-3095(94)90017-5.

33. Fisman, R.; Iyengar, S.S.; Kamenica, E.; Simonson, I. Gender Differences in Mate Selection: Evidence from a Speed Dating Experiment. Quarterly Journal of Economics 2006, 121, 673-697. doi:10.1162/qjec.2006.121.2.673.

34. Mortensen, D.T. The Matching Process as a Noncooperative Bargaining Game. In The Economics of Information and Uncertainty; National Bureau of Economic Research, 1982; Vol. I, pp. 173-203.

35. Bruch, E.E.; Newman, M.E.J. Aspirational Pursuit of Mates in Online Dating Markets. Science Advances 2018, 4, eaap9815. doi:10.1126/sciadv.aap9815.

36. Altman, I. Reciprocity of Interpersonal Exchange. Journal for the Theory of Social Behaviour 1973, 3, 249-261. doi:10.1111/j.14685914.1973.tb00325.x.

37. Tantardini, M.; Ieva, F.; Tajoli, L.; Piccardi, C. Comparing Methods for Comparing Networks. Scientific Reports 2019, 9, 1-19. doi:10.1038/s41598-019-53708-y.

38. Krackhardt, D. QAP Partialling as a Test of Spuriousness. Social Networks 1987, 9, 171-186. doi:10.1016/0378-8733(87)90012-8.

39. Mantel, N. The Detection of Disease Clustering and a Generalized Regression Approach. Cancer Research 1967, 27, $209-220$.

40. Dekker, D.; Krackhardt, D.; Snijders, T.A.B. Sensitivity of MRQAP Tests to Collinearity and Autocorrelation Conditions. Psychometrika 2007, 72, 563-581. doi:10.1007/s11336-007-9016-1.

41. Gode, D.K.; Sunder, S. Allocative Efficiency of Markets with Zero-Intelligence Traders: Market as a Partial Substitute for Individual Rationality. Journal of Political Economy 1993, 101, 119-137. doi:10.1086/261868.

42. Barabási, A.L.; Albert, R. Emergence of Scaling in Random Networks. Science 1999, 286, 509-512, [cond-mat/9910332]. doi:10.1126/science.286.5439.509.

43. Kreager, D.A.; Cavanagh, S.E.; Yen, J.; Yu, M. "Where Have All the Good Men Gone?" Gendered Interactions in Online Dating: Gendered Interactions in Online Dating. Journal of Marriage and Family 2014, 76, 387-410. doi:10.1111/jomf.12072.

44. Fiore, Andrew Rocco Tresolini, . Romantic Regressions: An Analysis of Behavior in Online Dating Systems. PhD thesis, 2004.

45. Hamilton, N.F. Romantic Relationships and Online Dating. In Applied Cyberpsychology; Attrill, A.; Fullwood, C., Eds.; Palgrave Macmillan UK: London, 2016; pp. 144-160. doi:10.1057/9781137517036_9.

46. Best, K.; Delmege, S. The Filtered Encounter: Online Dating and the Problem of Filtering through Excessive Information. Social Semiotics 2012, 22, 237-258. doi:10/gkqbkh.

47. Grazzini, J.; Richiardi, M.G.; Tsionas, M. Bayesian Estimation of Agent-Based Models. Journal of Economic Dynamics and Control 2017, 77, 26-47. doi:10.1016/j.jedc.2017.01.014.

48. Sumter, S.R.; Vandenbosch, L.; Ligtenberg, L. Love Me Tinder: Untangling Emerging Adults' Motivations for Using the Dating Application Tinder. Telematics and Informatics 2017, 34, 67-78. doi:10.1016/j.tele.2016.04.009. 